\title{
Poly-Arginine Peptide-18 (R18) Reduces Brain Injury and Improves Functional Outcomes in a Nonhuman Primate Stroke Model
}

\author{
Bruno P. Meloni ${ }^{1,2,3} \cdot$ Yining Chen ${ }^{4} \cdot$ Kathleen A. Harrison ${ }^{4}$ • Joseph Y. Nashed ${ }^{4}$. David J. Blacker ${ }^{1,2,5}$. \\ Samantha M. South ${ }^{6} \cdot$ Ryan S. Anderton ${ }^{1,3,7}$. Frank L. Mastaglia ${ }^{1,2} \cdot$ Andrew Winterborn $^{4} \cdot$ Neville W. Knuckey $^{1,2,3}$. \\ Douglas J. Cook ${ }^{4,8,9}$
}

Published online: 12 December 2019

(C) The American Society for Experimental NeuroTherapeutics, Inc. 2019

\begin{abstract}
Poly-arginine peptide-18 (R18) is neuroprotective in different rodent middle cerebral artery occlusion (MCAO) stroke models. In this study, we examined whether R18 treatment could reduce ischemic brain injury and improve functional outcome in a nonhuman primate (NHP) stroke model. A stroke was induced in male cynomolgus macaques by MCAO distal to the orbitofrontal branch of the MCA through a right pterional craniotomy, using a 5-mm titanium aneurysm clip for $90 \mathrm{~min}$. R18 $(1000 \mathrm{nmol} / \mathrm{kg})$ or saline vehicle was administered intravenously $60 \mathrm{~min}$ after the onset of MCAO. Magnetic resonance imaging (MRI; perfusion-weighted imaging, diffusion-weighted imaging, or T2-weighted imaging) of the brain was performed 15 min, $24 \mathrm{~h}$, and 28 days post-MCAO, and neurological outcome was assessed using the NHP stroke scale (NHPSS). Experimental endpoint was 28 days post-MCAO, treatments were randomized, and all procedures were performed blinded to treatment status. R18 treatment reduced infarct lesion volume by up to $65.2 \%$ and $69.7 \%$ at $24 \mathrm{~h}$ and 28 days poststroke, respectively. Based on NHPSS scores, R18-treated animals displayed reduced functional deficits. This study confirms the effectiveness of R18 in reducing the severity of ischemic brain injury and improving functional outcomes after stroke in a NHP model, and provides further support for its clinical development as a stroke neuroprotective therapeutic.
\end{abstract}

Key Words Stroke $\cdot$ poly-arginine peptide-18 $\cdot \mathrm{R} 18 \cdot$ neuroprotection $\cdot$ middle cerebral artery occlusion $\cdot$ nonhuman primate (macaque)

\section{Introduction}

The development of a clinically effective and safe neuroprotective drug for the treatment of ischemic stroke remains an

Electronic supplementary material The online version of this article (https://doi.org/10.1007/s13311-019-00809-1) contains supplementary material, which is available to authorized users.

Douglas J. Cook

dj.cook@queensu.ca

1 Perron Institute for Neurological and Translational Science, Nedlands, Western Australia 6009, Australia

2 Department of Neurosurgery, QEII Medical Centre, Sir Charles Gairdner Hospital, Nedlands, Western Australia, Australia

3 Centre for Neuromuscular and Neurological Disorders, The University of Western Australia, Perth, Western Australia, Australia

4 Centre for Neuroscience Studies, Queen's University, Kingston, Ontario, Canada urgent unmet need. Furthermore, given the demonstrated effectiveness of timely mechanical thrombectomy in large artery cerebral occlusion $[1,2]$, and the potential for an extended time window for alteplase thrombolysis in ischemic stroke
5 Department of Neurology, QEII Medical Centre, Sir Charles Gairdner Hospital, Nedlands, Western Australia, Australia

6 Office of Research Enterprise, The University of Western Australia, Perth, Western Australia, Australia

7 School of Heath Sciences, and Institute for Health Research, The University Notre Dame Australia, Fremantle, Australia

8 Division of Neurosurgery, Department of Surgery, Queen's University Kingston Health Sciences Centre, Kingston, Ontario, Canada

9 Division of Neurosurgery, Department of Surgery, Dalhousie University Halifax, Nova Scotia, Canada 
patients with a favorable perfusion image [3], neuroprotective therapies also have the potential to prolong the therapeutic window for reperfusion prior to endovascular interventions [4]. Such a therapy would preserve salvageable tissue in the penumbra, increasing the window in which recanalization can be achieved; decreasing tissue damage and potentially decreasing risk of hemorrhagic- and edema-related complications associated with prolonged ischemic times prior to reperfusion [5].

A series of studies from the Meloni laboratory have shown that the class of cationic arginine-rich peptides (CARPs) have potent neuroprotective properties [6-13]. In particular, poly-arginine-18 (R18; 18-mer of arginine; net charge +18 ) is a promising neuroprotective agent under development. R18 has been tested in in vitro and in vivo studies with strong neuroprotective efficacy observed in rodent models of middle cerebral artery occlusion [7, 9-12]. CARPs, including R18, are likely to possess a multimodal neuroprotective mechanism of action including the capacity to protect neurons from glutamate excitotoxicity and intracellular calcium influx [7], and reduce neuronal surface expression of the $N$-methyl-D-aspartate (NMDA) receptor subunit protein NR2B9c [14]. CARPs also reduce the activity and/or surface expression of other ion channels and receptors (e.g., AMPAR, TRPV1, NCX, CaV3.2, TNFR) that may exacerbate excitotoxicity following stroke [15-19]. This class of peptide also targets mitochondria and can reduce mitochondrial reactive oxygen species production and apoptosis $[20,21]$, scavenge free radicals $[22,23]$, inhibit the proteasome [24], and proprotein convertases that activate matrix metalloproteinases [25], modulate inflammatory responses [26, 27], and activate pro-cell survival signaling $[28,29]$.

Another more thoroughly characterized cationic peptide neuroprotectant, which is currently in phase III clinical trials $[30,31]$, is TAT-NR2B9c/NA-1 (HYGRKKRRQRRR-KLSSIESDV-OH; net charge +7). Tat-NR2B9c has demonstrated neuroprotective efficacy in multiple rodent [32-35] and nonhuman primate (NHP) [36, 37] stroke models, and following endovascular aneurysm repair procedures in a phase II human trial (ENACT trial) [38]. In a comparative study between R18 and Tat-NR2B9c using a model of transient middle cerebral artery occlusion (MCAO) in rats at a dose of $1000 \mathrm{nmol} / \mathrm{kg}$, R18 reduced infarct volume by $35.1 \%$ compared with a $26.1 \%$ reduction for Tat-NR2B9c [12].

Therefore, given the strong profile for neuroprotection of R18 from multiple rodent stroke models, the next step in the translational pipeline $[39,40]$ is to undertake a preclinical evaluation in a high-quality gyrencephalic NHP stroke model. In the present study, we examined the effectiveness of R18 following transient $\mathrm{MCAO}$ in the cynomolgus macaque.

\section{Material and Methods}

\section{R18 Peptide}

R18 (H-RRRRRRRRRRRRRRRRRR-OH) was synthesized to at least $98 \%$ purity, and subjected to peptide hydrolysis and amino acid liquid chromatography analysis to obtain a precise measure of peptide content (Mimotopes, Australia). The peptide was resuspended in $0.9 \%$ sodium chloride for injection, and $5 \mathrm{~mL}$ volumes transferred to $10 \mathrm{~mL}$ syringes and stored at $-20{ }^{\circ} \mathrm{C}$ until use.

\section{Blinding and Randomization Procedures}

A double-blinded approach was utilized for this study to maintain blinding of drug and placebo from technical staff, behavioral assessors, and imaging assessors. This was achieved by receiving lyophilized drug vials direct from the Meloni lab to the Queen's University facility. Then, a single technician, otherwise unrelated to the study, reconstituted all drug vials and randomly assigned a sequential identification number to drug and placebo vehicle ( $0.9 \%$ sodium chloride for injection) syringes that corresponded to the order of animal surgeries. The syringe assigned to the animal was confirmed by the principal investigator and one veterinary technician prior to administration. Syringe identification was recorded with animal identification at each step. Unblinding occurred after all experimental data had been collected and was performed by sending blinded data (i.e., animal identification and data) and the unblinding key (i.e., animal identification and treatment status) to an investigator not involved in the blinding process, treatment administration, or data collection.

\section{Animals and Stroke Model}

The study protocol was reviewed and approved by the Animal Use Subcommittee of Queen's University Council on Animal Care. All animal husbandry and experimental procedures were in conformance with the guidelines established by the Canadian Council for Animal Care (http://www.ccac.ca/en_/ standards/guidelines).

Animals were pair-housed prior to MCAO in home cages under controlled conditions with 12-h light/dark cycle, water ad libitum, and a daily diet in the form of Monkey Chow (Purina) and mixed dietary enrichment in the form of nuts, fresh fruit, and vegetables. Twenty healthy male captivebred cynomolgus macaques (Macaca fascicularis; $2.3-$ $2.7 \mathrm{~kg}$ ) underwent surgery for MCAO (R18, $N=10$ and saline, $N=10$ ) as previously described [36]. Briefly, animals fasted the evening prior to surgery were sedated using a mixture of ketamine $(5 \mathrm{mg} / \mathrm{kg})$ and dexmedetomidine 
$(0.05 \mathrm{mg} / \mathrm{kg})$, intubated and ventilated, and maintained by isoflurane anesthesia $\left(1.0-1.5 \% ; \mathrm{O}_{2}\right.$ flow rate of $\left.2 \mathrm{~L} / \mathrm{min}\right)$. Blood pressure (leg cuff) and body temperature (rectal probe) were monitored and maintained within normal physiological ranges during the procedure (Supplementary Table). Femoral arterial sampling was used intermittently to provide blood gases. MCAO in cynomolgus macaques was performed using a right pterional craniotomy and occluding the right MCA in the Sylvian fissure with a 5-mm titanium aneurysm clip distal to the orbitofrontal branch. Animals were transferred to the MRI 15 min after MCAO onset to confirm occlusion by perfusion and diffusion-weighted imaging. At the end of the 90min duration of MCAO, the aneurysm clip was removed in order to restore blood flow. Animal body temperature was maintained $\left(37 \pm 0.5^{\circ} \mathrm{C}\right)$ throughout the surgery and MRI scanning by heating blanket.

R18 (1000 nmol/ $\mathrm{kg})$ or saline vehicle was infused (1 $\mathrm{mL} / \mathrm{kg}$ over $10 \mathrm{~min}$ ) intravenously to animals through the saphenous vein, $60 \mathrm{~min}$ after the onset of MCAO. This timing was chosen to mimic an optimal human stroke treatment scenario, in which treatment is delayed from occlusion onset to presentation at a medical facility, followed by rapid reperfusion. In addition, the dose of R18 was selected based on a previous dose-response study performed in the Meloni laboratory using a rat model of transient MCAO demonstrating high efficacy at $1000 \mathrm{nmol} / \mathrm{kg}$ [12].

Postsurgery, animals were individually placed in clean cages, continually monitored until fully recovered from anesthesia and allowed free access to food and water. Once fully recovered, animals were returned to their home cages and housed as described above. Animal exclusion criteria consisted of nonreperfusion following reversal of MCAO, significant weight loss ( $>20 \%$ presurgery weight), continuous seizure, or severe disability requiring continuous care for basic functions.

\section{MRI Acquisition}

Magnetic resonance scanning was performed at the Queen's University Centre for Neuroscience Studies using a $3 \mathrm{~T}$ Siemens Trio scanner with a 32-channel head coil. For the acquisition of MRI images, the animals were intubated and anesthetized (isoflurane 1.0-1.5\%) throughout the scanning. Baseline MRI acquisitions (perfusion-weighted imaging (PWI) and diffusion-weighted imaging (DWI)) were made $15 \mathrm{~min}$ after MCAO, and poststroke MRI acquisitions (DWI and T2-weighted imaging (T2WI)) were obtained $24 \mathrm{~h}$ and 28 days post-MCAO.

All images were acquired in DICOM format and converted to NIfTI format using dcm2nii from MRIcron [41]. NIfTI files were imported to analysis software for segmentation and volumetric measurements.

\section{Lesion Volume Analysis}

Methods for volumetric analysis have been described previously [36]. Briefly, NIfTI image stacks were imported to ITKSNAP software for semi-automated segmentation and automated contouring [42]. User-guided 3D active contour segmentation of anatomical structures significantly improved efficiency and reliability. Perfusion volumes were measured using cerebral blood flow maps in which lesional tissue was defined as having a flow less than $20 \mathrm{cc} / 100 \mathrm{~g} / \mathrm{min}$; DWI and T2WI volumes were measured by segmenting and contouring region of hyperintensity in the MCA territory.

\section{Neurological Assessment}

Neurological outcomes were monitored using the nonhuman primate stroke scale (NHPSS) as previously validated and described for this stroke model [36, 43]. The NHPSS score consists of an 11-part assessment of neurological function comprising state of consciousness, defense reaction, grasp reflex, extremity movement, gait, circling, bradykinesia, balance, neglect, visual field cut/hemianopsia, and facial weakness. From a total of 41 points, 0 corresponds to normal behavior and 41 to severe bilateral neurological impairment. Previous experiments in macaques subjected to a 90-min MCAO typically show an initial peak in NHPSS scores that persisted for the first $36 \mathrm{~h}$ and then gradually improved over time [36].

\section{Statistical Analyses}

Ischemic lesion volume measurements (DWI and T2WI) for the R18 treatment group was compared with the vehicle control group by one-way analysis of variance (StatView; version 4.51, Abacus Concepts Inc., Berkeley, CA, USA). At each time point, data from the NHPSS was analyzed using the Mann-Whitney $U$ test (StatView). A linear mixed model (LMM) was used to investigate the longitudinal NHPSS data, as this analysis is able to account for repeated measures for each animal (SPSS Statistics for Windows; version 25, IBM Corp., Armonk, NY, USA). A Bonferroni correction factor was applied with the LMM and adjusted $p$ values are reported. The experiment group (R18 or vehicle) and time (days) were included in the linear mixed model, with dependent variable outcome being the composite NHPSS score. A residual check for normality as per the LMM assumption was conducted on the final models. A value of $p<0.05$ was considered significant for all data sets. Data in the figures are presented as mean \pm standard error (SE). Sample size ( $N=10$; i.e., intention-totreat) was calculated based on previous data for the stroke model [36] and a 40\% treatment effect (infarct volume reduction), power $80 \%$, and alpha level $=0.05$. 


\section{Results}

\section{Animal Exclusions and Baseline Data}

In the 24- to 36-h acute poststroke phase, two animals died; one saline-treated (due to edema), and one R18-treated (due to seizure) that also did not reperfuse. In addition, two other R18treated animals did not reperfuse and were excluded from the study. No other animals exhibited significant weight loss or disability requiring additional care. Final sample size consisted of nine saline- and seven R18-treated animals.

Perfusion (PWI) and diffusion (DWI) imaging $15 \mathrm{~min}$ after MCAO to quantify the tissue at risk and identify initial lesion development, respectively, did not differ in R18 and vehicle treatment animals (Figs. 1a and 2).

\section{Infarct Lesion Outcomes}

At 24-h poststroke, based on DWI, R18 reduced infarct lesion volume by $61.5 \%(p=0.0002)$ (Figs. $1 \mathrm{~b}$ and 2$)$. Similarly, at 28 days poststroke, based on T2WI, R18 reduced infarct lesion volume by $66.6 \%(p<0.0001)$ (Figs. $1 \mathrm{c}$ and 2$)$. Furthermore, to account for infarct variability after MCAO, the infarct volume of each animal was normalized to its MRI perfusion deficit (i.e., tissue at risk) measured $15 \mathrm{~min}$ after MCAO. Normalization to tissue at risk revealed that R18 at $24 \mathrm{~h}$ and 28 days poststroke reduced infarct lesion volume by $65.2 \%(p=0.0003)$ and $69.7 \%(p<0.0001)$, respectively (Fig. 1d, e). Based on DWI and T2WI, there was no evidence of hemorrhagic transformation in saline- and R18-treated animals.

\section{Functional Outcome}

The R18 treatment group recorded a lower NHPSS score compared with the vehicle treatment group at all poststroke time points (Fig. 3). When observing repeated measures over the course of the 28-day poststroke period, the linear mixed model analysis revealed a significant reduction of 2.682 points in the NHPSS in the R18-treated group when compared with vehicle $(p=0.002$; Table 1). For each unit of time (day), a 0.343 point reduction in the NHPSS was observed $(p<0.001)$, indicating a greater improvement in functional deficits in R18-treated animals over the 28 days after stroke. Based on the MannWhitney analysis, differences in the NHPSS on any individual poststroke time point were not statistically significant.

\section{Discussion}

This study provides further validation of the neuroprotective properties of R18 after stroke, and highlights its potential as an acute ischemic stroke therapeutic, particularly in the setting of
Perfusion and diffusion: 15 min post-MCAO

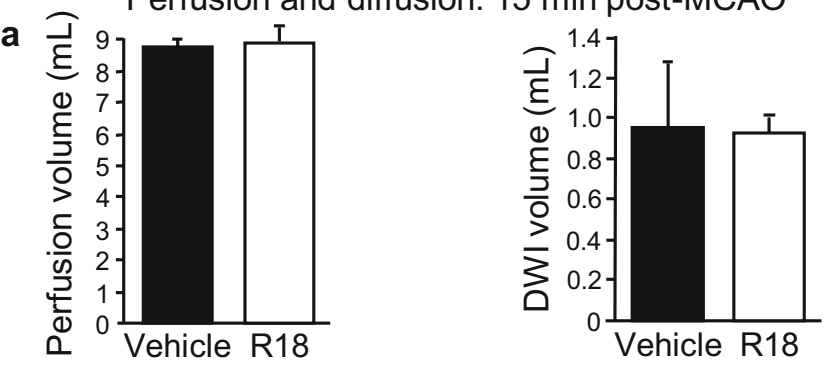

Infarct lesion: $24 \mathrm{~h}$ post-stroke
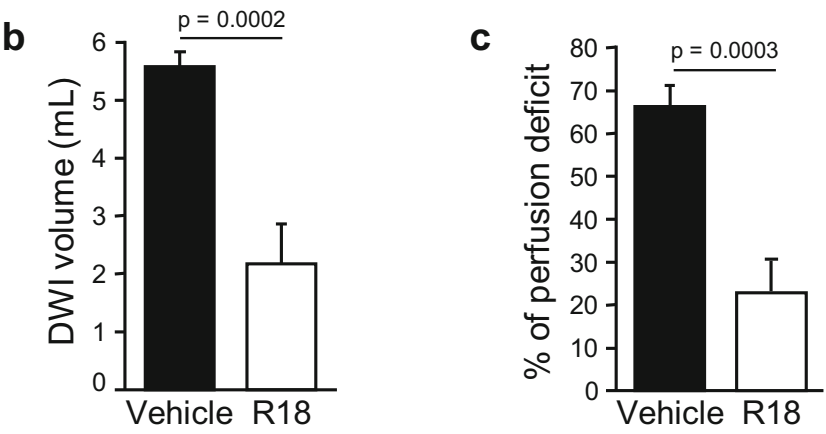

Infarct lesion: 28 d post-stroke d

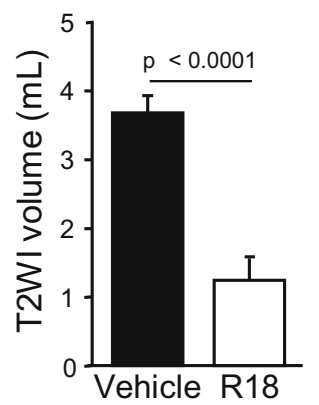

e

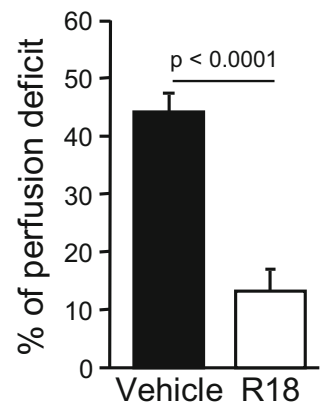

Fig. 1 MRI imaging data for R18 (1000 nmol/kg) and saline vehicle treatment groups at different time points after middle cerebral artery occlusion (MCAO). (a) Perfusion volume deficits and DWI lesion volumes $15 \mathrm{~min}$ after MCAO. (b) Stroke lesion volumes as measured by DWI $24 \mathrm{~h}$ after MCAO. (c) Stroke lesion volumes as measured by DWI normalized to the initial perfusion deficit for each animal $24 \mathrm{~h}$ after MCAO. (d) Stroke lesion volumes as measured by T2WI 28 days after MCAO. (e) Stroke lesion volumes as measured by T2WI and normalized to the initial perfusion deficit for each animal 28 days after MCAO. $N=7$ for $\mathrm{R} 18$ and $N=9$ for saline. Values are means $\pm \mathrm{SE}$

large vessel occlusion and recanalization. For example, this and our previous studies have demonstrated that R18 has the capacity to reduce infarct volume, cerebral hemisphere swelling and functional deficits in several different rodent stroke models (permanent and transient MCAO, and ET-1-induced MCAO), in different rat strains (Sprague-Dawley, Wistar) and now in a NHP (macaque) [9-12, 44].

In this study, treatment with R18 was highly effective in reducing infarct volume at both $24 \mathrm{~h}$ and 28 days after stroke, confirming that the neuroprotective effects are maintained, and also improved functional outcomes. Moreover, as 
Fig. 2 Representative MRI images of perfusion deficits and stroke lesions obtained in R18$(1000 \mathrm{nmol} / \mathrm{kg})$ and saline vehicle-treated animals $15 \mathrm{~min}$, $24 \mathrm{~h}$, or 28 days after middle cerebral artery occlusion
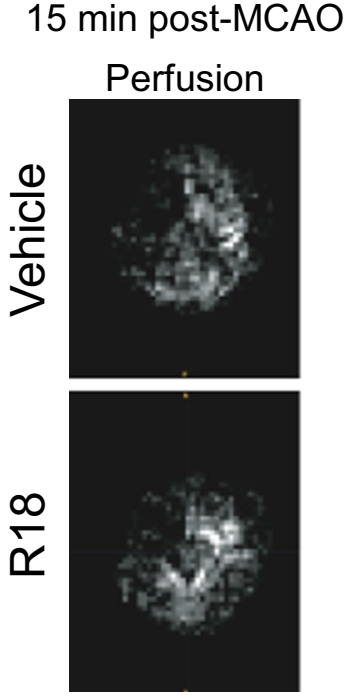

$24 \mathrm{~h}$ post-stroke

DWI
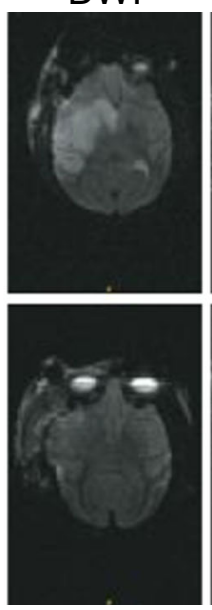

T2WI
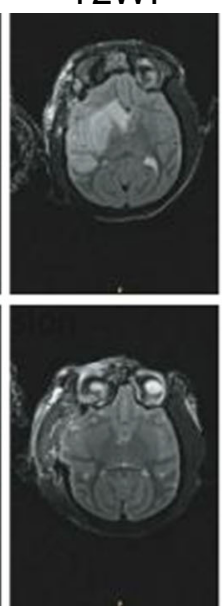

28 d post-stroke
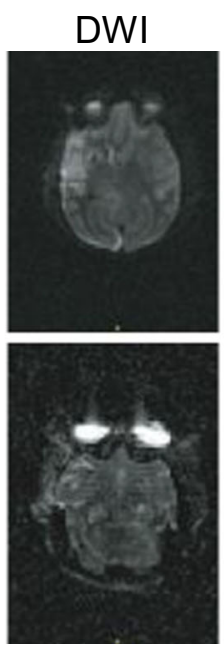

T2WI

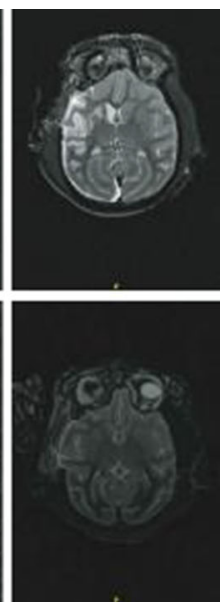

treatment was commenced 60 min after stroke onset and before reperfusion, this suggests that the peptide would have the capability of extending the therapeutic time window for thrombolysis and endovascular thrombectomy after a thromboembolic stroke.

Whereas this study did not investigate neuroprotective mechanisms of action, we and others have previously demonstrated that R18 and other CARPs are likely to have a multimodal mechanism of action. For example, R18 has been shown to reduce excitotoxic neuronal intracellular calcium influx [7] and to reduce inflammatory responses, caspase activation, and autophagy following traumatic brain injury $[45,46]$, whereas shorter polyarginine peptides such as R4, R7, or R9 can assist in maintaining mitochondrial integrity during oxidative and calcium-induced stress [20, 21, 47], inhibit matrix metalloproteinase activation [25], and provide cellular protection from hydrogen peroxide injury [48]. However, we

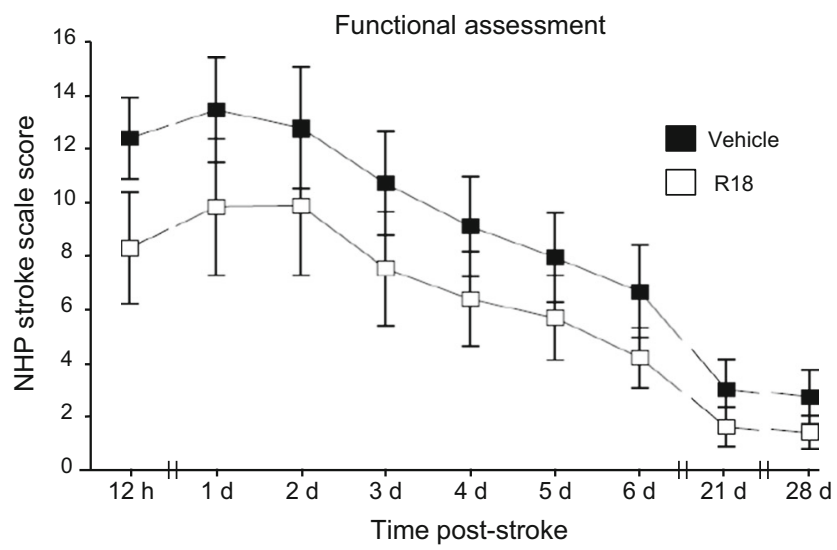

Fig. 3 Nonhuman primate stroke scale (NHPSS) scores for R18 $(1000 \mathrm{nmol} / \mathrm{kg})$ and saline vehicle treatment groups at different time points after middle cerebral artery occlusion. $N=7$ for R18 and $N=9$ for saline. Values are mean scores $\pm \mathrm{SE}$ acknowledge that further studies are required to confirm these mechanisms of action and basis for the neuroprotective effects of R18 in vivo in the NHP stroke model.

In addition, we did not investigate R18 uptake into the brain or other pharmacodynamic and pharmacokinetic aspects of the peptide distribution and metabolism following intravenous administration. However, with respect to brain uptake, it is known that CARPs can cross the blood-brain barrier $[49,50]$ and are often used as carriers to deliver other molecular cargoes (e.g., proteins, peptides, nucleic acids) into the CNS [51, 52]. For example, in the case of the NA-1 peptide, the cationic arginine-rich TAT peptide (YGRKKRRQRRR) is used as a carrier molecule [32]. Furthermore, following intravenous administration in mice, the poly-arginine-11 peptide (R11) enters the intact brain, and brain uptake is further enhanced when administered after MCAO [53]. Therefore, based on the high neuroprotective efficacy of R18 observed in this study and the known capacity of CARPs to enter the brain, it is likely that R18 does enter the brain, particularly in areas in which the blood-brain barrier is compromised. Thus, it would be of interest for future studies to determine R18 brain tissue concentrations in healthy animals and in animals after stroke, as well as determine concentrations of the peptide that provide maximum

Table 1 Linear mixed model parameter estimates for total NHPSS with experimental group as the independent variable

\begin{tabular}{lclll}
\hline Variable & Coefficient & Standard error & $t$ & $p$ \\
\hline Intercept & 8.915 & 0.729 & 12.232 & $<0.001$ \\
Vehicle & 2.682 & 0.844 & 3.178 & 0.002 \\
R18 & $0^{*}$ & - & - & - \\
Time & -0.343 & 0.046 & -7.526 & $<0.001$ \\
\hline
\end{tabular}

*Indicates comparison group 
therapeutic effect. In this regard, whereas the $1000 \mathrm{nmol} /$ $\mathrm{kg}$ dose of R18 was selected based on a dose-response study performed in rats [12], the optimal dose of R18 in the macaque and in humans may in fact be significantly lower, as extrapolating intravenous doses from small animals (e.g., rats) to larger animals (e.g., humans) should be based on body surface area [54], rather than weight. On this basis, the $1000 \mathrm{nmol} / \mathrm{kg}$ dose in the rat would be equivalent to a $325 \mathrm{nmol} / \mathrm{kg}$ and $165 \mathrm{nmol} / \mathrm{kg}$ dose in the macaque and an adult human, respectively. Importantly, in humans, a lower therapeutic dose of R18 would have the advantage of minimizing any potential adverse side effects of the peptide.

Based on the serum pharmacokinetics of the CARP protamine (PRRRRSSSRPVRRRRRPRVSRRRRRRGGRRRR; net charge +21), the serum half-life of free R18 peptide is likely to be in the order of 7-24 $\min [55,56]$. In support of this, in a pharmacokinetic study performed in our laboratory using R18D (D-enantiomer of R18), free peptide was detectable by HPLC-mass spectrometry 5-min postintravenous infusion, but not at $10 \mathrm{~min}$ or later (unpublished data). However, in contrast to free peptide, R18 is likely to bind to serum proteins such as albumin, with the effect of extending its serum half-life for several hours, as has been demonstrated for R9D/ALX40-4C (D-enantiomer of R9: Ac-rrrrrrrr- $\mathrm{NH}_{2}$; net charge +9) [57]. This is likely to be significant from a therapeutic standpoint, as protein bound R18 provides a reservoir of the peptide to exert its neuroprotective actions over an extended time period, which would be highly beneficial in ischaemic stroke because brain injury can proceed over many hours. Therefore, future preclinical serum pharmacokinetic studies with R18 should examine both free and protein bound peptide.

Whereas analysis of the NHPSS data using a linear mixed model to investigate the longitudinal improvement in neurological deficits confirmed a highly significant R18 treatment effect, improvement at different time points poststroke appeared to be relatively modest. This is most likely because of the smaller than expected ischemic lesions, resulting in milder functional deficits associated with this model, and consequently a reduced sensitivity of the NHPSS to detect treatment effects. Whereas the exact reasons for the milder strokes observed in this study are not known, it is noteworthy that the animals were smaller and younger, and obtained from a different source to those used in previous studies in our laboratory. Furthermore, the recovery in functional deficits observed in both R18- and vehicle-treated animals by day 21 poststroke most likely reflects their greater capacity for plasticity and to regain lost function after stroke, compared with aged animals. Therefore, additional studies will be needed to confirm that R18 can sustain long-term functional benefits in older animals.

\section{Conclusion}

This study has confirmed the capacity of R18 to reduce brain injury and improve functional outcomes following MCAO in the macaque stroke model, and paves way for further development of the peptide as a potential standalone stroke therapeutic or an adjunct treatment to extend the therapeutic time window for endovascular interventions, particularly after large artery occlusion ischemic stroke. Whereas experience to date with the CARP group of peptides including NA-1 [38], ALX40-4C [57], and CN-105 (Ac-VSRRR-NH ${ }_{2}$; net charge +3 ) [58] has not identified any significant toxicity issues, more intensive pharmacokinetic and safety studies of $\mathrm{R} 18$ will be a prerequisite prior to consideration of evaluation in clinical stroke trials.

Acknowledgments This work was supported by funding provided by the Multiple Sclerosis Society of Western Australia (MSWA) and a private donation from Helen Sewell (AM).

\section{Compliance with Ethical Standards}

The study protocol was reviewed and approved by the Animal Use Subcommittee of Queen's University Council on Animal Care. All animal husbandry and experimental procedures were in conformance with the guidelines established by the Canadian Council for Animal Care (http://www.ccac.ca/en_/standards/guidelines).

Conflict of Interest Bruno Meloni and Neville Knuckey are the holders of several patents regarding the use of cationic arginine-rich peptides as neuroprotective treatments. Samantha South is employed within the Office of Research Enterprise, The University of Western Australia, which oversees the intellectual property and commercial development concerning R18. The other authors declare no conflict of interest.

\section{References}

1. Rabinstein AA. Stroke in 2015: acute endovascular recanalization therapy comes of age. Nat Rev Neurol. 2016; 12:67-8.

2. Nogueira RG, Jadhav AP, Haussen DC, Bonafe A, Budzik RF, Bhuva P, et al. DAWN trial investigators. Thrombectomy 6 to 24 hours after stroke with a mismatch between deficit and infarct. $\mathrm{N}$ Engl J Med. 2018; 378:11-21.

3. Campbell BCV, Ma H, Ringleb PA, Parsons MW, Churilov L, Bendszus $M$, et al. EXTEND, ECASS-4, and EPITHET Investigators. Lancet. 2019; 394:139-47.

4. Savitz SI, Baron JC, Yenari MA, Sanossian N, Fisher M. Reconsidering neuroprotection in the reperfusion era. Stroke. 2017; 48:3413-19.

5. Thomalla G, Sobesky J, Köhrmann M, Fiebach JB, Fiehler J, Zaro Weber O, et al. Two tales: hemorrhagic transformation but not parenchymal hemorrhage after thrombolysis is related to severity and duration of ischemia: MRI study of acute stroke patients treated with intravenous tissue plasminogen activator within 6 hours. Stroke. 2007; 38:313-18.

6. Meloni BP, Craig AJ, Milech N, Hopkins RM, Watt PM, Knuckey NW. The neuroprotective efficacy of cell-penetrating peptides TAT, penetratin, Arg-9, and Pep-1 in glutamic acid, kainic acid, and in vitro ischemia injury models using primary cortical neuronal cultures. Cell Mol Neurobiol. 2014; 34, 173-81. 
7. Meloni BP, Brookes LM, Clark VW, Cross JL, Edwards AB, Anderton RS. et al. Poly-arginine and arginine-rich peptides are neuroprotective in stroke models. J Cereb Blood Flow Metab. 2015; 35:993-1004.

8. Meloni BP, Milani D, Edwards AB, Anderton RS, O'Hare Doig RL, Fitzgerald M. Neuroprotective peptides fused to arginine-rich cell penetrating peptides: Neuroprotective mechanism likely mediated by peptide endocytic properties. Pharmacol Ther. 2015; 153 : 36-54.

9. Meloni BP, South SM, Gill DA, Marriott AL, Déziel RA, Jacques A. et al. Poly-arginine peptides R18 and R18D improve functional outcomes after endothelin-1-induced stroke in the Sprague Dawley rat. J Neuropathol Exp Neurol. 2019; 426-35.

10. Milani D, Clark VW, Cross JL, Anderton RS, Knuckey NW, Meloni BP. Poly-arginine peptides reduce infarct volume in a permanent middle cerebral artery rat stroke model. BMC Neurosci. 2016; 17, 19.

11. Milani D, Knuckey NW, Cross JL, Anderton RS, Meloni BP. The R18 polyarginine peptide is more effective than the TAT-NR2B9c (NA-1) peptide when administered 60 minutes after permanent middle cerebral artery occlusion in the rat. Stroke Res Treat. 2016; 2016:1-9.

12. Milani D, Cross JL, Anderton RS, Blacker DJ, Knuckey NW, Meloni BP. Neuroprotective efficacy of R18 poly-arginine and NA-1 (TAT-NR2B9c) peptides following transient middle cerebral artery occlusion in the rat. Neurosci Res. 2017; 114:9-15.

13. Edwards AB, Cross JL, Anderton RS, Knuckey NW, Meloni BP. Poly-arginine R18 and R18D (D-enantiomer) peptides reduce infarct volume and improves behavioural outcomes following perinatal hypoxic-ischaemic encephalopathy in the P7 rat. Mol. Brain. 2018; 11, 1-12.

14. MacDougall G, Anderton RS, Edwards AB, Knuckey NW, Meloni BP. The neuroprotective peptide poly-arginine-12 (R12) reduces cell surface levels of NMDA NR2B receptor subunit in cortical neurons; investigation into the involvement of endocytic mechanisms. J Mol Neurosci. 2018; 61:235-46.

15. Ferrer-Montiel AV, Merino JM, Blondelle SE, Perez-Payà E, Houghten RA, Montal M. Selected peptides targeted to the NMDA receptor channel protect neurons from excitotoxic death. Nat Biotechnol. 1998; 16:286-91.

16. Brustovetsky T, Pellman JJ, Yang XF, Khanna R, Brustovetsky N. Collapsin response mediator protein 2 (CRMP2) interacts with Nmethyl-D-aspartate (NMDA) receptor and $\mathrm{Na}^{+} / \mathrm{Ca}^{2+}$ exchanger and regulates their functional activity. J Biol Chem. 2014; 289:7470-82.

17. Planells-Cases R, Aracil A, Merino JM, Gallar J, Pérez-Payá E, Belmonte C, et al. Arginine-rich peptides are blockers of VR-1 channels with analgesic activity. FEBS Lett. 2000; 481:131-6.

18. García-Caballero A, Gadotti VM, Stemkowski P, Weiss N, Souza IA, Hodgkinson V, et al. The deubiquitinating enzyme USP5 modulates neuropathic and inflammatory pain by enhancing Cav3.2 channel activity. Neuron. 2014; 83:1144-58.

19. Fotin-Mleczek M, Welte S, Mader O, Duchardt F, Fischer R, Hufnagel $\mathrm{H}$, et al. Cationic cell-penetrating peptides interfere with TNF signalling by induction of TNF receptor internalization. J Cell Sci. 2005; 118:3339-51.

20. Marshall J, Wong KY, Rupasinghe CN, Tiwari R, Zhao X, Berberoglu ED, et al. Inhibition of N-methyl-D-aspartate-induced retinal neuronal death by polyarginine peptides is linked to the attenuation of stress-induced hyperpolarization of the inner mitochondrial membrane potential. J Biol Chem. 2015; 290:22030-48.

21. MacDougall G, Anderton RS, Mastaglia FL, Knuckey NW, Meloni BP. Mitochondria and neuroprotection in stroke: cationic argininerich peptides (CARPs) as a novel class of mitochondria-targeted neuroprotective therapeutics. Neurobiol Dis. 2019; 121:17-33.

22. Kown MH, Lijkwan MA, Jahncke CL, Murata S, Rothbard JB, Robbins RC. L-Arginine polymers enhance coronary flow and reduce oxidative stress following cardiac transplantation in rats. $\mathrm{J}$ Thorac Cardiovasc Surg. 2003; 126:1065-70.

23. Mandal SM, Bharti R, Porto WF, Gauri SS, Mandal M, Franco OL, et al. Identification of multifunctional peptides from human milk. Peptides. 2014; 56:84-93.

24. Gaczynska M, Osmulski PA, Gao Y, Post MJ, Simons M. Prolineand arginine-rich peptides constitute a novel class of allosteric inhibitors of proteasome activity. Biochemistry. 2003; 42:8663-70.

25. Cameron A, Appel J, Houghten RA, Lindberg I. Polyarginines are potent furin inhibitors. J Biol Chem. 2000; 275:36741-9.

26. Hilchie AL, Wuerth $\mathrm{K}$, Hancock RE. Immune modulation by multifaceted cationic host defense (antimicrobial) peptides. Nat Chem Biol. 2013; 9:761-8.

27. Li LH, Ju TC, Hsieh CY, Dong WC, Chen WT, Hua KF, et al. A synthetic cationic antimicrobial peptide inhibits inflammatory response and the NLRP3 inflammasome by neutralizing LPS and ATP. PLoS One. 2017; 12:e182057.

28. Gu Q, Zhai L, Feng X, Chen J, Miao Z, Ren L, et al. Apelin-36, a potent peptide, protects against ischemic brain injury by activating the PI3K/Akt pathway. Neurochem Int. 2013; 63:535-40.

29. Yang Y, Zhang XJ, Li LT, Cui HY, Zhang C, Zhu CH, et al. Apelin13 protects against apoptosis by activating AMP-activated protein kinase pathway in ischemia stroke. Peptides. 2016; 75:96-100.

30. Field Randomization of NA-1 Therapy in Early Responders (FRONTIER). http://www.strokecenter.org/trials/clinicalstudies/ field-randomization-of-na-1-therapy-in-early-responders/ description.

31. Safety and Efficacy of NA-1 in Subjects Undergoing Endovascular Thrombectomy for Stroke (ESCAPE-NA1). http://www. strokecenter.org/trials/clinicalstudies/safety-and-efficacy-of-na-1in-subjects-undergoing-endovascular-thrombectomy-for-strokeescape-na1/description.

32. Aarts M, Liu Y, Liu L, Besshoh S, Arundine M, Gurd JW. et al. Treatment of ischemic brain damage by perturbing NMDA receptor-PSD-95 protein interactions. Science. 2002; 298:846-50.

33. Bratane BT, Cui H, Cook DJ, Bouley J, Tymianski M, Fisher M. Neuroprotection by freezing ischemic penumbra evolution without cerebral blood flow augmentation with a postsynaptic density-95 protein inhibitor. Stroke. 2011; 42:3265-70.

34. Soriano, FX, Martel, MA, Papadia, S, Vaslin, A, Baxter, P, Rickman, C, et al. Specific targeting of pro-death NMDA receptor signals with differing reliance on the NR2B PDZ ligand. J Neurosci. 2008; 28:10696-710.

35. Sun HS, Doucette, TA, Liu, Y, Fang, Y, Teves, L, Aarts, M. Effectiveness of PSD95 inhibitors in permanent and transient focal ischemia in the rat. Stroke. 2008; 39:2544-53.

36. Cook DJ, Teves L, Tymianski M. Treatment of stroke with a PSD95 inhibitor in the gyrencephalic primate brain. Nature. 2012; 483: 213-17.

37. Cook DJ, Teves L, Tymianski M. A translational paradigm for the preclinical evaluation of the stroke neuroprotectant Tat-NR2B9c in gyrencephalic nonhuman primates. Sci Transl Med. 2012; 4: $154 \mathrm{ra} 133$.

38. Hill MD, Martin RH, Mikulis D, Wong JH, Silver FL, Terbrugge $\mathrm{KG}$, et al. ENACT trial investigators. Safety and efficacy of NA-1 in patients with iatrogenic stroke after endovascular aneurysm repair (ENACT): a phase 2, randomised, double-blind, placebocontrolled trial. Lancet Neurol. 2012; 11:942-50.

39. Cook DJ, Tymianski M. Nonhuman primate models of stroke for translational neuroprotection research. Neurotherapeutics. 2012; 9: 371-79.

40. Bosetti F, Koenig JI, Ayata C, Back SA, Becker K, Broderick JP, et al. Translational stroke research: vision and opportunities. Stroke. 2017; 48:2632-37.

41. Rorden C, Brett M. Stereotaxic display of brain lesions. Behav Neurol. 2000; 12:191-200. 
42. Yushkevich PA, Piven J, Hazlett HC, Smith RG, Ho S, Gee JC, et al. User-guided 3D active contour segmentation of anatomical structures: significantly improved efficiency and reliability. Neuroimage. 2006; 31:1116-28.

43. Roitberg B, Khan N, Tuccar E, Kompoliti K, Chu Y, Alperin N, et al. Chronic ischemic stroke model in cynomolgus monkeys: behavioral, neuroimaging and anatomical study. Neurol Res. 2003; 25:68-78.

44. Milani D, Bakeberg MC, Cross JL, Clark VW, Anderton, RS, Blacker DJ, et al. Comparison of neuroprotective efficacy of poly-arginine R18 and R18D (D-enantiomer) peptides following permanent middle cerebral artery occlusion in the Wistar rat and in vitro toxicity studies. Plos One. 2018; 13:e0193884.

45. Chiu LS, Anderton RS, Cross JL, Clark VW, Knuckey NW, Meloni BP. Poly-arginine peptide R18D reduces neuroinflammation and functional deficits following traumatic brain injury in the LongEvans rat. Int J Peptide Res Ther. 2019; https://doi.org/10.1007/ s10989-018-09799-8.

46. Batulu H, Du G-J, Li D-Z, Sailike D, Fan Y-H, Geng D. Effect of poly-arginine R18 on neurocyte cell growth via autophagy in traumatic brain injury. Exp Ther Med. 2019; 17: 4109-15.

47. Rigobello MP, Barzon E, Marin O, Bindoli A. Effect of polycation peptides on mitochondrial permeability transition. Biochem Biophys Res Commun. 1995; 217:144-9.

48. Fonar G, Polis B, Meirson T, Maltsev A, Samson AO. Subcutaneous sustained-release of poly-arginine ameliorates cognitive impairment in a transgenic mouse model of Alzheimer's disease. Adv Alzheimer's Dis. 2018; 7:153-82.

49. Stalmans S, Bracke N, Wynendaele E, Gevaert B, Peremans K, Burvenich $\mathrm{C}$, et al. Cell-penetrating peptides selectively cross the blood-brain barrier in vivo. PLoS One. 2015; 10:e0139652.

50. Pham W, Zhao BQ, Lo EH, Medarova Z, Rosen B, Moore A. Crossing the blood-brain barrier: a potential application of myristoylated polyarginine for in vivo neuroimaging. Neuroimage. 2005; 28:287-92.

51. Cai SR, Xu G, Becker-Hapak M, Ma M, Dowdy SF, McLeod HL. The kinetics and tissue distribution of protein transduction in mice. Eur J Pharm Sci. 2006; 27:311-19.

52. Zou LL, Ma JL, Wang T, Yang TB, Liu CB. Cell-penetrating peptide-mediated therapeutic molecule delivery into the central nervous system. Curr Neuropharmacol. 2013; 11:197-208.

53. Gotanda Y, Wei FY, Harada H, Ohta K, Nakamura KI, Tomizawa $\mathrm{K}$, et al. Efficient transduction of 11 poly-arginine peptide in an ischemic lesion of mouse brain. J Stroke Cerebrovasc Dis. 2014; 23:2023-30.

54. Nair AB, Jacob $\mathrm{S}$. A simple practice guide for dose conversion between animals and human. J Basic Clin Pharm. 2016; 7:27-31.

55. DeLucia A 3rd, Wakefield TW, Kadell AM, Wrobleski SK, VanDort M, Stanley JC. Tissue distribution, circulating half-life, and excretion of intravenously administered protamine sulfate. ASAIO J. 1993; 39:M715-18.

56. Butterworth J, Lin YA, Prielipp R, Bennett J, James R. The pharmacokinetics and cardiovascular effects of a single intravenous dose of protamine in normal volunteers. Anesth Analg. 2002; 94: 514-22.

57. Doranz BJ, Filion LG, Diaz-Mitoma F, Sitar DS, Sahai J, Baribaud F, et al. Safe use of the CXCR4 inhibitor ALX40-4C in humans. AIDS Res Hum Retroviruses. 2001; 17:475-86.

58. Guptill JT, Raja SM, Boakye-Agyeman F, Noveck R, Ramey S, Tu TM, et al. Phase 1 randomized, double-blind, placebo-controlled study to determine the safety, tolerability, and pharmacokinetics of a single escalating dose and repeated doses of $\mathrm{CN}-105$ in healthy adult subjects. J Clin Pharmacol. 2017; 57:770-6.

Publisher's Note Springer Nature remains neutral with regard to jurisdictional claims in published maps and institutional affiliations. 